\title{
Resultados preliminares del estudio de seguimiento de egresados de la Licenciatura en Intervención Educativa, líneas específicas: educación inicial y educación inclusiva, de la Universidad Pedagógica Nacional del Estado de Chihuahua, Unidad Parral
}

\author{
Carlos Alfonso Valenzuela Maldonado \\ Universidad Pedagógica Nacional del Estado de Chihuahua \\ Unidad Parral \\ carlos alfonsov@hotmail.com \\ José Luis Rodríguez Canales \\ Universidad Pedagógica Nacional del Estado de Chihuahua \\ Unidad Parral \\ joseluisrodri48@hotmail.com \\ Gerardo Roacho Payán \\ Universidad Pedagógica Nacional del Estado de Chihuahua \\ Unidad Parral \\ groacho@gmail.com
}

\section{Resumen}

El estudio surge de la necesidad de información que permita analizar la función educativa de la Universidad reflejada en el papel que los egresados de la LIE actualmente desempeñan en la sociedad; así mismo de tener una visión del desarrollo y tendencias de las líneas profesionalizantes; además, poder contar con un directorio de egresados que posibilite su localización. En consecuencia, se inicia un estudio de corte mixto con prioridad cuantitativo y ejecución secuencial de corte descriptivo - transversal para observar, describir y presentar en dos momentos la opinión de los egresados desde diferentes ámbitos recabados mediante el análisis de documentos y expedientes institucionales, así como la aplicación de un instrumento de recopilación mixto compuesto de preguntas abiertas y cerradas de tipo escala de opinión previamente diseñado, piloteado y sustentado bajo la metodología sugerida por ANUIES (2003), todo lo anterior con el objetivo de: a) conocer la opinión de los egresados acerca de la formación académica recibida y sobre su proceso de integración al mundo laboral; y, b) constituir un banco de datos y materiales de información acerca de los egresados. Se inicia la aplicación del instrumento con egresados del año 2009, muestra compuesta por 43 personas, de las cuales se hace contacto con 36 , quince alumnos de educación inclusiva y veintiuno de educación inicial, los datos recabados se concentraron en una base de datos diseñada con SPSS, lo cual ha permitido el análisis, descripción y 
presentación de la información, así como la extracción del directorio de contacto de egresados.

\author{
Palabras clave \\ Seguimiento, egresados, LIE.
}

\section{Introducción}

La Universidad Pedagógica Nacional del Estado de Chihuahua, está viviendo un proceso de cambio radical en su organización, administración, gestión y procesos universitarios, lo cual ha permitido que en unos cuantos meses, haya emprendido una serie trabajos a favor del mejoramiento y transformación. Entre los proyectos que la Universidad se ha propuesto llevar a cabo, son los estudios de seguimiento de egresados para los programas de Maestría en Educación Básica (MEB) y Licenciatura en Intervención Educativa (LIE).

En este sentido abordaremos los avances que para el programa de la LIE existen, para lo cual hemos definido un apartado para la definición del problema de estudio, el cual ha sido sustentado desde la importancia de los estudios de seguimiento de egresados; así mismo, se hacen necesarios desde la perspectiva de los requerimientos de organizaciones e instituciones que se encargan de la evaluación de las instituciones de educación superior; finalmente se plantea la necesidad de contar con un directorio que permita una comunicación más eficiente con los egresados.

Posteriormente se enuncian los objetivos que guiaron el proceso de la investigación y mediante los cuales se ha diseñado el proceso metodológico, el cual viene enunciado en el apartado III y que está compuesto por el tipo y diseño de la investigación, así como de la descripción del instrumentos utilizado para la recopilación de la información; así mismo, se describe el proceso de selección de la muestra; para finalmente, hacer referencia al diseño de la base de datos que nos permitió cristalización de los datos para iniciar con la redacción del reporte preliminar del estudio de seguimiento de egresados.

Posteriormente, se realiza un análisis de las variables de mayor relevancia según los objetivos que hemos definido al inicio de proceso.

\section{Problema de estudio.}

Los estudios de seguimientos de egresados representan una necesidad que permiten recabar, analizar y presentar información de trascendental importancia en los procesos de gestión institucional, sobre todo a lo que tomas de decisiones se refiere. "La evaluación de las actividades de las IES encuentra en los estudios de egresados una fuente de información vital, pues a partir de ellos la institución se autoanaliza en conexión con su entorno" (Valenti y Varela, 2003, p.32)

Así mismo, representan uno de los requerimientos no menos importantes de instituciones y programas como ANUIES, FOMES y CIEES, ya que permiten tener una visión clara del desarrollo y tendencias de las líneas profesionalizantes de la Licenciatura en Intervención Educativa (LIE).

Por otro lado, las Instituciones de Educación Superior (IES), requieren de un contacto efectivo que permita mantener una relación de cooperación mutua con los 
egresados, con el fin de adquirir las experiencias y recabar las necesidades que desde el ámbito laboral puedan aportar a la Universidad.

Por lo anteriormente anotado, se plantea la necesidad de iniciar con un estudio de seguimiento de egresados para la LIE, líneas profesionalizantes educación inicial e inclusiva, el cual brinde los elementos necesario para establecer criterios de mejora continua en los procesos académicos que se llevan a cabo en la institución, así como también permita la construcción de un directorio de egresados de la Institución.

\section{Objetivos}

Los fines que se persiguen en un primer momento se dirigen en 2 direcciones:

Evaluar el impacto de la tarea educativa emitida por la Universidad Pedagógica Nacional del Estado de Chihuahua (UPNECH), Unidad Parral, en la formación de interventores educativos, tomando en cuenta la atención recibida durante su estancia en la UPNECH y el contexto laboral en el que actualmente se desempeñan y;

Construir un directorio con información de egresados.

\section{Consideraciones Metodológicas.}

Se realiza un diseño de investigación de corte mixto con prioridad cuantitativo y ejecución secuencial de corte descriptivo longitudinal, ya que: "los métodos mixtos representan un conjunto de procesos sistemáticos, empíricos y críticos de investigación e implican la recolección y el análisis de datos cuantitativos y cualitativos, así como su integración y discusión conjunta”. (Hernández, 2010, p.546)

El diseño anterior permitió recabar, analizar, interpretar y presentar de una manera sistematizada la opinión de los egresados desde los diferentes ámbitos recabados mediante el análisis de documentos institucionales e instrumento compuesto de preguntas abiertas y escalas de opinión previamente diseñado, piloteado, modificado y sustentado bajo la metodología sugerida por ANUIES.

Cabe mencionar que el instrumento está compuesto por diez módulos los cuales se enuncian a continuación: módulo de datos generales, de estudios en la institución, continuación de la formación, empleo durante los estudios, búsqueda de empleo al egresar de la institución, empleo actual, opinión sobre la formación recibida y sobre la institución, escolaridad y ocupación del padre o jefe de familia, actualización de directorio y valoración; en su conjunto conforman un total de 84 preguntas.

La tarea de recopilación de la información, se realizará en dos mementos, ya que así es sugerido por diversos investigadores. "El estudio de seguimiento de egresados en seguir longitudinalmente a los egresados y entrevistarlos al menos en dos momentos posteriores al egreso. La experiencia muestra la conveniencia de que la primera encuesta se realice al menos tres años después de que egresaron y la segunda se realice entre el quinto y sexto años posteriores a la misma fecha", (Valenti y Varela, 2003, p.26).

Por otro lado, "en un estudio longitudinal a la población objeto de estudio se le consulta en varios momentos de su proceso de inserción laboral. Un buen parámetro para la comparación de resultados es el contar con al menos tres aplicaciones de la encuesta a lo largo del tiempo previsto" (Ramos, 2006, p. 32); también encontramos que: “...la observación de las características de los egresados de la carrera de licenciatura se estudian los mismos sujetos, pero, en por 
lo menos, dos momentos distintos..." (Ontiveros, 2006, p.12)

Se elige iniciar la aplicación del instrumento con egresados del año 2009, muestra conformada por 43 personas, de las cuales se hace contacto con 36 , quince de educación inclusiva y veintiuno de educación inicial.

Para el proceso de recopilación de la información se consideró a los alumnos becarios quienes mediante entrevistas y medios tecnológicos - electrónicos recuperaran la información ya que en algunos casos se hizo contacto con egresados que se encontrabas incluso fuera del estado.

Mientras tanto se diseñó la base de datos con el programa SPSS, lo cual ha permitido el análisis, descripción y presentación de la información que ha posibilitado la elaboración del reporte de resultados preliminares.

\section{Resultados.}

\section{A. Población}

La población estudiada corresponde a la generación 2005-2009, en donde se puede percibir que el sexo masculino es de un $33.3 \%$, mientras el $66.7 \%$ son mujeres, se infiere que está es una carrera predominantemente feminista, por el porcentaje considerable de mujeres universitarias egresadas, como se puede apreciar es el doble.

La edad de las y los encuestados fluctúa al momento del egreso entre los 22-40 años, se observa que sólo un $21.2 \%$ de jóvenes ingresaron a la carrera en tiempos correspondientes a la secuencia de sus estudios, es decir, sin interrupción y un $9 \%$ corresponden a personas de la población adulta y el $91 \%$ son jóvenes.

El estado civil mayoritario son solteras (as) con un porcentaje del 54.5, esto les facilitó dedicarle tiempo a sus estudios, mientras un $24.2 \%$ casadas las dificultades eran más notorias por la condición de su estado civil, asimismo los padres/madres solteras que constituyen un $6.1 \%$, el resto presenta diferentes situaciones, entre ellos, divorciadas, viudas y unión libre.

Los y las egresadas en las dos líneas; Inclusiva e Inicial, ofertadas en la institución, hubo una preferencia del 60.6\% hacia la línea de Inicial, y en la línea de Inclusión un 39.4\%, reflejando promedios con una media de 9.25 , en el rango desde un 8.06 a 9.93, siendo los de mayor frecuencia: 9.0, 9.4 y 9.8.

\section{B. Competencias Generales}

Los alumnos egresados de ambas líneas están formados bajo las siguientes competencias generales profesionales: crear ambientes de aprendizaje, realizar diagnósticos socioeducativos, diseñar programas y proyectos pertinentes para ámbitos formales y no formales, asesorar a individuos, grupos e instituciones, planear procesos, acciones y proyectos educativos, identificar, desarrollar y adecuar proyectos educativos, evaluar instituciones, procesos y sujetos y desarrollar procesos de formación permanente.

Tomando en cuenta las categorías más seleccionadas o donde más se concentraron las respuestas, los egresados opinaron sobre la relación existente entre las competencias y las actividades que desarrollan en sus ámbitos laborales:

1.- Crear ambientes de aprendizaje:

De 36 egresados 19 expresaron con una frecuencia de "siempre", tener la experiencia de hacer uso de esta competencia en sus ámbitos laborales, lo cual representa un $48.7 \%$ de la muestra entrevistada.

$$
\text { 2.- Realizar diagnósticos }
$$


socioeducativos:

De la muestra entrevistada, un total de 17 egresados manifestaron que siempre han utilizado o puesto en práctica la presente competencia, lo cual representa un $47.2 \%$ de los egresados entrevistados.

3.- Diseñar programas y proyectos pertinentes para ámbitos formales y no formales:

En la aplicación y desempeño de la presente competencia profesional, 13 de los 36 entrevistados, expresaron realizar esta actividad, lo cual viene a representar un $36.1 \%$ de la muestra que participo.

4.- Asesorar a individuos, grupos e instituciones:

De los 36 egresados que emitieron su respuesta, 15 aceptaron realizar esta actividad de una manera ordinaria, lo cual viene a representar un $41.6 \%$ de la muestra a la que se le consulto.

5.- Planear procesos, acciones y proyectos educativos:

En esta competencia profesional, un total de 24 egresados manifestaron realizarla de manera cotidiana, tomando en cuenta que la muestra está compuesta por 36 personas, viene a representar el $66.6 \%$.

6.- Identificar, desarrollar y adecuar proyectos educativos:

En lo que se refiere a las actividades en donde se pone de manifiesto la presente competencia profesional, de 36 entrevistados 18 respondieron realizarla de manera cotidiana, lo que representa un $50 \%$ de la muestra.

7.- Evaluar instituciones, procesos y sujetos:

De la misma manera el $50 \%$ de la muestra entrevistada, afirmaron poner en práctica la presente competencia profesional de una manera cotidiana.

8.- Desarrollar procesos de formación permanente:
Finalmente, 18 de 36 entrevistados afirmaron poner en práctica las actividades que hacen uso de la presente competencia, lo cual representa un $50 \%$ de la muestra.

\section{Eficiencia terminal}

La eficiencia terminal se considera muy alta, debido a que el $97 \%$ está titulado(a), y de estos el $78.8 \%$ lo hizo de manera inmediata, con una opción de tesis del 97\%, contemplando está forma de titulación como la más formativa con un $33.3 \%$, mientras $48.4 \%$ lo percibe como lo más fácil, accesible y por ser el procedimiento establecido respectivamente.

\section{Empleo actual}

Nuestros ex estudiantes están empleados en un $97 \%$, siendo solo un $3 \%$ que no lo tiene.

La necesidad de entrar al campo laboral es importante, para ello, el 69\% lo consiguió en menos de 3 meses, mientras que el $21.2 \%$ lo logró entre 3 a 6 meses, el $3.0 \%$ que tardó de 6 meses a un año, quedando que el $6.1 \%$ tardó más de un año.

Se desempeña un $97.8 \%$ en el sector Público, ante un $15.2 \%$ en el sector privado y solo un $3.0 \%$ se desempeña de manera independiente, de tal manera que el ramo donde tienen su quehacer profesional es en Educación en un 97\% del total de alumnos.

En cuanto a los medios que utilizaron para conseguir el empleo, el $39 \%$ fue por iniciativa propia, mientras que el $30 \%$ por medio de Convocatorias, cabe resaltar que por recomendación fue el $18.2 \%$. Finalmente el $6.1 \%$ a partir de la Bolsa de empleo y el $3.0 \%$ fue en el periódico.

\section{Conclusiones}

Una vez que se han presentado una parte 
de los resultados que pueden ser extraídos gracias a la aplicación del instrumento de recopilación de la información y sobre todo de la base de datos diseñada para concentrar la información, podemos decir que actualmente la UPNECH, Unidad Parral, cuenta con información científica que le facilite la toma de decisiones en diferentes ámbitos como lo son: capacitación, docencia, contratación, impacto, factibilidad de la líneas, entre otras; así mismo cuenta con un punto de vista externo sobre los procesos educativos que en la Universidad se llevan a cabo, por lo que pueden ser evaluados para emprender estrategias o acciones en favor.

Por otro lado, se inició la concentración de la información generada por el modulo I (datos generales) y IX (actualización de directorio) con el fin de contar con un directorio de egresados que permita la búsqueda para la participación, cooperación y mutuo apoyo entre la Universidad y los egresados de la misma.

\section{Referencias}

Hernández, R. (2010) Metodología de la Investigación. McGraw Hill. Quinta edición. México.

Ontiveros, I. (2006) Seguimiento de Egresados de la Licenciatura en Artes Visuales de la Escuela de Pintura, Escultura y Artesanías de la UJED. UPD. México.

Ramos, T. (2006) Manual de Instrumentos y Recomendaciones sobre el Seguimiento de Egresados. ALFA. México.

Valenti, G., Varela, G. (2003) Diagnóstico sobre el estado actual de los estudios de egresados. México. ANUIES. 